\title{
The Challenges of Multidisciplinary Education in Computer Science
}

\author{
Fred S. Roberts \\ DIMACS Center, Rutgers University, Jersey, New Brunswick, NJ, U.S.A.
}

E-mail: froberts@dimacs.rutgers.edu

Received December 24, 2010; revised June 21, 2011.

\begin{abstract}
Some of the most important problems facing the United States and China, indeed facing our entire planet, require approaches that are fundamentally multidisciplinary in nature. Many of those require skills in computer science (CS), basic understanding of another discipline, and the ability to apply the skills in one discipline to the problems of another. Modern training in computer science needs to prepare students to work in other disciplines or to work on multidisciplinary problems. What do we do to prepare them for a multidisciplinary world when there are already too many things we want to teach them about computer science? This paper describes successful examples of multidisciplinary education at the interface between CS and the biological sciences, as well as other examples involving CS and security, CS and sustainability, and CS and the social and economic sciences. It then discusses general principles for multidisciplinary education of computer scientists.
\end{abstract}

Keywords multidisciplinary, biology, sustainability, security, social sciences, economics, education

\section{The Challenge: Computer Science in Service to Society}

Computer science (CS) is increasingly connected to areas outside the discipline. Modern training in CS needs to prepare students to work with those in other disciplines or to work on multidisciplinary problems. But we already have too many things we want to teach them about computer science. What do we do to prepare them for a multidisciplinary world? This is an increasingly important challenge facing us as we seek ways to educate the next generation of computer scientists and prepare them to work in a rapidly-changing world.

Some of the most important problems facing the United States and China, indeed facing our entire planet, require approaches that are fundamentally multidisciplinary in nature. Many of those require skills in computer science, basic understanding of another discipline, and the ability to apply the skills in one discipline to the problems of another. Protection of our natural environment is a case in point, where analysis of changing climates requires sophisticated computer science models, but understanding the impact of climate change on life on the planet requires fundamental understanding of ecosystems and species and their dependence on temperature, moisture, etc., as well as the impact of changing climatic conditions on mosquitoes and other carriers of diseases (e.g., malaria).
Modern approaches to health care depend critically on understanding genetics, and modern genetics has become fundamentally an information science, depending in crucial ways on the development of methods of computer science to understand molecular sequences, protein function, etc. At the same time, the health of our people can be greatly enhanced by the involvement of computer scientists in overcoming challenging problems involving the use of electronic medical records and in the use of information technology to enable the treatment of patients from a distance, in both cases requiring a true understanding of how modern medical science works.

In the area of security, some of the most critical challenges to our societies arise from sophisticated weapons of a biological nature such as engineered pathogens (e.g., smallpox) as well as naturally-occurring, but newly-devastating diseases such as pandemic influenza and SARS. Other challenges require the ability to apply powerful methods of machine learning and artificial intelligence to get early warning of an impending natural disaster or a terrorist plot.

As we recognize that our dependence on traditional sources of energy may cause both environmental and economic challenges, due to unwanted side effects of the use of oil or coal for example and due to looming shortages, computer scientists can help to develop energy-efficient architectures, energy-efficient network protocols, "smart metering" systems, and energy-aware 
communication and task scheduling. They can also help us to develop models that will enable us to use computing tools in a more energy-efficient way and models that will help us determine how to dispatch power from intermittent sources such as wind. To make these contributions, computer scientists will need to understand the economics of energy, the alternative sources of energy (both traditional and nontraditional), and become versed in the interplay among energy, environment, and economics.

Computer scientists have an important role to play in helping our increasingly-interconnected world economies remain healthy, by developing tools to enhance and protect ubiquitous digitized economic transactions and to model the potential outcomes of new economic policies concerning credit, trade, incentives, etc. To do so, computer scientists will need to develop fundamental understanding of today's complex financial institutions.

Approaches to critical problems of society will require development of a workforce that is well prepared to understand the interconnections among disciplines. To function in this rapidly changing world, students of computer science will have to gain access to the most important foundational and emerging concepts and processes at the interface between their discipline and others, and will need to develop a coherent and increasingly sophisticated understanding of the ways that two disciplines interact, the new knowledge that is created through this interaction, and the new educational and career opportunities open to them in the increasingly multidisciplinary world. There has never been a more crucial time than now to ensure that we train the next generation of computer scientists to be able to think broadly across disciplines. If we succeed in doing this training well, we can involve computer science and computer scientists in a fundamental way in addressing some of the most challenging problems facing society in the United States, China, and the rest of the world.

However, how can we do this without sacrificing their training in their own discipline? Moreover, without knowing which of many other disciplines an individual might come in contact with in the future, how can we train computer science students to interact with disciplines that they might not even encounter while they are in school? These issues confront both U.S. and Chinese computer scientists and are the frequent subject of discussion among and between them.

\section{Getting to Them When They Are Young: Examples from the Biological Sciences}

It is critical to begin the introduction to multidisciplinary experiences when students are young. A case in point is the interface between the biological sciences on the one hand and mathematical sciences, including computer science, on the other hand. We have already seen a critically important role for CS in connection with the biological sciences. The human genome project is a case in point. It reflects the fact that biology is increasingly becoming an information science. The study of biomathematics and computational biology has become important in graduate education and increasingly in undergraduate education. However, the high schools have been slow to pick it up. At our research center, DIMACS, we have found that the interface between biology and math/CS can be introduced with great success even at the high school level. We can take away some insights from the DIMACS program.

In the summer of 2004, we instituted the DIMACS BioMath Connect Institute (BMCI) (see http://dimacs.rutgers.edu/dci/2005/). We brought to our center high school mathematics teachers (who were teaching mathematics and computer science). We introduced them to the field of computational molecular biology during the course of a week of introductory lectures followed by a week in which they were working on problems arising from this topic, doing research under the guidance of a mentor. These teachers brought their experiences back to their classrooms. In the United States, the high school curriculum is very crowded. There is no room to introduce lengthy new units into courses. Thus, our teachers concentrated on short units of one week or less. The teachers brought back research problems for students to work on, emulating the experiences they had had at DIMACS. The response to this approach by both teachers and students was very enthusiastic.

In Phase II of the DIMACS BMCI program, we added biology teachers and brought high school math/computer science and biology teachers to our center together. We put emphasis on teams of teachers from the same school, one math/CS teacher and one biology teacher. We gave math/CS teachers a short tutorial on relevant biology and biology teachers a short tutorial on relevant math/CS, and then introduced the teachers, sitting together, to computational molecular biology. The teachers helped their colleagues from the opposite discipline to understand the lectures and worked on problems together. We then had some of the teachers join in interdisciplinary groups to work on research questions and others work on writing materials that could be used in their classrooms. The teachers took their experiences back to their classrooms, taught short units of a week or less, often in "teams" who went together to each others' classrooms, and engaged the students. The response, as before, was considerable enthusiasm on the part of the teachers and students. In 
later years, we expanded the scope of the program to introduce different topics, topics from computational and mathematical epidemiology and from ecology and population biology.

The BMCI program put an emphasis on teacher training. But teacher training is not sufficient. We can only reach a miniscule percentage of teachers by bringing small groups of them together to get exposure to new ideas. On the other hand, new materials that teachers and students can use can reach many people and are absolutely essential if we are to introduce them to topics at the interface between disciplines. Thus, in the Fall of 2006, we introduced a new program, the DIMACS BioMath Connection (BMC) (see http://dimacs.rutgers.edu/BMC/). This program has been developing 15 "modules" to be used in high school math/CS and biology classes. The modules are, once again, aimed at short periods of a week or less, and they have been tested out in classrooms and evaluated by professional evaluators, aiming at understanding their impact on both the students and the teachers who use them. The topics have been chosen from computational molecular biology, computational and mathematical epidemiology, and ecology and population biology.

For example, the BMC module "Spider Silk" asks students to apply knowledge of protein structure and function to pose and answer the fundamental question: What alignment of two sequences is biologically most meaningful? The module develops the basic mathematical principles that underlie computer programs used to align amino acids nearly instantaneously. After becoming familiar with spiders, their webs and their silks, students use graphs (networks), dynamic programming, and recursive thinking to model sequence alignments. To give another example, the module "Mathematical Modeling of Disease Outbreaks" introduces simple mathematical models that can answer some of the following questions: Will there be a flu outbreak this season? How many individuals will become infected? How long will it persist? Would vaccination prevent an epidemic? What other measures could be taken to prevent an epidemic?

Our evaluation has aimed at testing several theses:

- Exposing biology students to the importance of mathematics and computer science methods in biology (including computational thinking/algorithms) will help them appreciate biology more.

- Exposing mathematics/CS students to the usefulness of mathematical sciences methods in modern biological problems will help them appreciate mathematics/CS more.

- Exposing students to the interface between the biological and mathematical sciences will open up new horizons for them and expose them to new career opportunities and new opportunities for further education.

- Exposing students to the interface between the biological and mathematical sciences will motivate them as students.

Extensive evaluation has found support for all four theses.

We have recently initiated a fourth phase of our high school program, "The Challenges of Interdisciplinary Education: Math-Bio" (IMB). In IMB, we are expanding the topical coverage of BMC, including new modules in such areas as neuroscience, biological communication (e.g., cellular signaling), biological timing/"biological clock" functions, biological feedback systems, and medical imaging. We are also designing several full courses that will incorporate the modules from BMC and the new ones, and will be preparing a book consisting of the relevant modules, chosen from the BMC modules and the new ones. This is a departure from our previous emphasis on short units that can be inserted into existing courses, but reflects the increasingly-sophisticated interconnections between math/CS and the biological sciences.

For more on the topic of biology/CS in the high schools, see Cozzens and Roberts ${ }^{[1]}$ and, in particular, the articles by Cozzens ${ }^{[2]}$ and by Roberts ${ }^{[3]}$ in that book.

\section{Examples from Other Disciplines}

The CS-biology interface is only one example. We have done similar things with other areas, even for middle schools. For example, our center for "homeland security" at DIMACS (CCICADA - the Command, Control and Interoperability Center for Advanced Data Analysis) has introduced math/CS middle school and high school teachers and students to algorithms for inspection of containers at ports, location of sensors for nuclear detection, and analysis of data to give early warning of an outbreak of a new disease. We ran a tutorial on these topics for high school teachers and have worked with them to find ways to implement these topics in the classroom. We also have a program of module writing as part of this activity. An early module for middle school students involved assignment of police officers to different areas where crime is most heavy. This included development of modifications of the algorithms for solving assignment problems. New modules are being prepared on applications of cryptography to homeland security, on the applications of generalized ideas about tomography to problems of food safety and security, and on the location of surveillance centers for foot and mouth disease. There has been a very positive response to the entire topic of surveillance for early 
warning of serious events, whether natural disasters (hurricanes, tornadoes, tsunamis), disease events (such as the outbreak of new strains of influenza), or terrorist events.

The growing human population and increasing pressures for development have led to a variety of challenges for life on our planet, in particular whether current patterns of human activity are sustainable. Human activity is closely tied to the natural environment and there is a two-way interconnection between human activity and environmental processes. Increasingly, we are noting how human activities affect the systems that sustain life, including climate, healthy air and water, availability of food. The earth has finite resources that we need to sustain our life style: sources of energy, clean water, arable land. As environmental conditions change, there are possibilities for new diseases, species can move into areas to which they are non-native and crowd out the species to which we are accustomed, and the human condition can be threatened by environmental change. Fundamental societal structures such as national boundaries and the health of our economic systems can be affected by competition for changing natural resources, shortages and in some cases surpluses. These problems are complex, multi-disciplinary, and intertwined and do not respect international borders. They call for a "science of sustainability" [4]. These problems are natural for US-China collaboration since sustainability problems do not stop at national boundaries, and the mutual interest in these problems was reflected in an emphasis on this topic in a series of China-US Computer Science Leadership Summits held in 2006, 2008, and 2010.

Education in CS and sustainability is reflected in the various activities of Cornell University's Institute of Computational Sustainability (http://www.cis.cornell.edu/ics/). Research challenges in sustainability are closely related to educational opportunities, and they are reflected in the report on "Mathematical and Statistical Challenges for Sustainability" [5] that arose from a workshop held at DIMACS in November 2010 and a report on "The Role of Information Sciences and Engineering in Sustainability" [6] that arose from a workshop sponsored by the US National Science Foundation (NSF) in February 2011, and will be reflected in a workshop on US-China Collaborations in CS and Sustainability to be held at DIMACS in September 2011. They are also reflected in a new "Sustainable Planet Education Program" we are launching at DIMACS in connection with Mathematics of Planet Earth (MPE), a program sponsored by 14 North American Mathematical Sciences Research Institutes in conjunction with many international organizations and professional societies (see http://www.mpe2013.org/). A key mission of MPE is to increase the engagement of mathematical scientists (including computer scientists and including researchers, teachers, students, and the public) with issues of planet Earth, and in particular creating a sustainable environment and economic system. Encouraging teachers at all levels to create and use planetrelated curriculum materials is among the key aspects of MPE. As part of MPE, DIMACS, in collaboration with the Mathematics and Climate Research Network based at Bowdoin College, is leading the development of MPE-related modules for the undergraduate level, which instructors can bring into their classrooms highlighting mathematical sciences topics associated with MPE. MPE module themes will include sustainable resource management, the interface between sustainability and economic systems, climate change, disaster response, control of diseases and epidemics, energy production and utilization, uncertainty quantification and decision support, among others. Possible topics for modules include natural resource bioeconomics, with sensitivity to economic discount rate and resource spatial heterogeneity; "cradle to grave" life cycle assessment of energy production, consumer products, food production and transportation; land use economics in a changing climate; critical transitions and resilience in simple and complex models (e.g., aquatic systems and water quality); energy balance models of climate, climate sensitivity and climate feedback processes; fossil fuels and atmospheric composition; data assimilation in chaotic systems (e.g., weather); efficient evacuation of major metropolitan areas susceptible to increased flooding; measuring biodiversity and resilience of ecosystems with decreasing biodiversity (e.g., 1840's Irish potato blight); determining optimal strategies for utilizing various electrical energy generating stations given uncertainties about wind energy and solar energy; and presentation of knowledge and uncertainty for effective decision support ${ }^{1}$.

The connections between CS and the social sciences are not as well developed as those between CS and the biological sciences. However, these connections presage a "new frontier" for computer science. The importance of this emerging frontier is reflected in the development of a new NSF program entitled "Interface between Computer Science and Economics and Social Science." Many applications in computer science involve issues and problems that social scientists have

\footnotetext{
(1) The author thanks Midge Cozzens, Eugene Fiorini and Mary Lou Zeeman for this list of potential topics and for some of the language describing the Sustainable Planet Education Project.
} 
addressed for years, issues of preference, utility, conflict and cooperation, allocation, incentives, consensus, social choice, and measurement. We have begun to see the use of methods developed by social scientists in a variety of computer science applications. The requirements posed by these computer science applications place great strain on the social science methods because of the sheer size of the problems addressed, new contexts in which computational power of agents becomes an issue, limitations on information possessed by players, and the sequential nature of repeated applications. Hence, there is a great need to develop a new generation of methods to satisfy these computer science requirements. In turn, these new methods will provide powerful new tools for social scientists. (For a survey article about these new "social-science-based" computer science methodologies and their application to problems of computer science and of the social sciences, see [7].) Important topics in this area include metasearch, collaborative filtering, and recommender systems; computational tractability/intractability of consensus functions; algorithmic issues in game theory; computational issues in auction design; market clearing algorithms; and algorithmic decision theory (the latter the topic of an international conference held in Venice in $2009^{[8]}$ — and to be held again in 2011 at DIMACS). A very interesting example of a course that reflects these kinds of themes is a course called Networks that is offered at Cornell University and listed jointly as a course in the departments of Economics, Sociology, Computer Science and Information Science (see http://www.infosci.cornell.edu/courses/info2040. 2010 sp/ for a course description of the Spring 2010 version of the course). This course is offered at the introductory undergraduate level and is based on a book coauthored by a computer scientist and an economist ${ }^{[9]}$, who have team-taught the course. Topics covered include graph theory and social networks, game theory, markets and strategic interaction on networks, information networks and the World Wide Web, network dynamics (both population models and structural models), and institutions and aggregate behavior.

A number of "CS and social sciences" modules are being developed in a DIMACS project called "The Value of Computational Thinking Across Grade Levels 9 to 12" (VCTAL). This project is developing, testing, and implementing an innovative mix of 12 instructional modules for grades 9 to 12 (and sometimes beyond), with student and teacher versions and one-page "teasers" for each module, and packaging of the modules with suggested scope and sequence for a one-semester course. The project is also working with students and teachers to determine the influence of these materials on increasing interest of diverse students in computer science and other technical fields. One interesting planned module is on power indices: How do you determine the power of an individual in an organization, a state in a US presidential election, or a symptom in determining a disease? The module introduces various weighted voting systems and poses ways of determining the power of each player (person, state, symptom) when each player has a different number of votes (weights), including examples of Corporate Boards of Directors where there are few players and the US presidential electoral college where there are 51 players. The topics include the need for efficient algorithms to calculate various power indices. A second planned module in VCTAL deals with the "tragedy of the commons." The classic version of this refers to a pasture that is held in common by $N$ herdsmen. The common pasture can support a fixed number $G$ of cattle, so that each herdsman can keep $G / N$ cattle on the common ground. But a herdsman wants to increase his/her number of cattle to increase his/her profit, and others will then want to do likewise until eventually the pasture is destroyed. As discussed in its classic formulation, each herdsman is a "rational maximizer" and is immune from anything but overt coercion. This module looks at what makes most real-life commons robust and self-stabilizing. Students are encouraged to develop these reasons, which include psychologicallydetermined utility functions, and a variety of different cooperative strategies. Students explore individual and group social values and behaviors in the context of achieving sustainable resources. Simulations and modeling become important tools used in the student explorations. This module is appropriate for the first two years of college or advanced high school students in mathematics, psychology, sociology, and computer science classes.

\section{Some General Principles}

While many of these examples come from programs for precollege students, there are some general principles that arise from them that are applicable more generally to multidisciplinary education for computer scientists at all levels. Here are some relevant principles that have come out of our experiences and that could help in designing ways to overcome the challenges we face:

- Start them young.

- It does not take a full course. Small units can have 
great impact.

- "Instructor training" helps.

- Creating links between instructors in different disciplines helps (team teaching, joint preparation, etc.).

- Research experiences for students, even very junior students, help.

- Appropriate materials need to be developed.

- "One size does not fit all".

So, how do we prepare computer science students to understand other disciplines, work on problems arising in other disciplines, and work with people in such disciplines? There are a variety of approaches. One approach is to introduce "units" in existing courses, units which reflect the connection between CS and another discipline. As we have observed, these do not have to be long. Another approach is to introduce specialized courses where there is enough material for such a course. Computational molecular biology, computational economics, and networks are cases in point. A third approach is to introduce "case study" seminars or courses. These are more practically oriented, and often include guest lecturers from other disciplines or industry.

Team experiences can simulate the increasingly important emphasis on working in multidisciplinary teams, but the team experience in a course (such as a team project) does not have to be multidisciplinary to introduce students to the best techniques for working within a team. This is a way of preparing students to work in another discipline by giving them skills that are, at least in principle, independent of what that other discipline is. An interesting example of the team approach to multidisciplinary education is the Berkeley Lab Computing Sciences "Berkeley CSE" program (see http://www.lbl.gov/cs/cse/index.html\#vision). This program "provides a framework for enhanced support of collaborative research projects involving CSE researchers and students from its three organizational partners." Faculty from 20 programs are participating in Berkeley CSE, ranging from Astronomy to Chemistry to Materials Science to Political Science.

Other important ways to prepare CS students to work with other disciplines is to arrange internships for them, e.g., in industry or government laboratories. For undergraduates in the U.S., we have a very successful program called REU, Research Experiences for Undergraduates. There is considerable evidence that exposing undergraduates to research experiences can be a crucial factor in getting them to continue their education in graduate school. Increasingly, these research experiences can be multidisciplinary. At DIMACS, for example, we bring in close to 50 undergraduate students each year, chosen from applicants from around the country (and in some cases from partners in other countries). They work one on one with a mentor. In other REU programs, the students often work in teams. There are many successful models for REU. Over the years, we have increasingly had students work on multidisciplinary projects in our REU. For instance, in recent years, we have had students work on mining EEG data to detect epilepsy; protein-induced DNA looping; mathematical modeling to simulate HIV outbreaks in the third world and understand the interplay between disease spread and allocation of monetary resources to different health interventions; algorithms to identify and evacuate at-risk individuals during heat events due to global warming; container inspection algorithms in ports; using biometrics to detect botnet attacks; and the influence of economic aspects on disease-avoiding behavior. Our experience is that students take easily to learning what they need to learn in another discipline in order to do real research projects even while still undergraduates. It is complicated enough to do a research project within one's own discipline as an undergraduate, but, interestingly, the challenges of working on problems from another discipline and seeing the value of their training in computer science contributes to students doing very successful multidisciplinary projects. Virtually $100 \%$ of our REU students go on to graduate school, many point to the undergraduate research experience as having influenced them to do so, and many build on their multidisciplinary projects as undergraduates to do multidisciplinary work for their Masters or Ph.D. degrees. In this way, an early multidisciplinary experience can lead to an interest in multidisciplinary work in the future.

In all of these cases, introducing CS students to work in other disciplines is perhaps more important than the particular discipline outside CS. It does not have to be any particular discipline outside CS so much as providing early exposure with a multidisciplinary experience.

It can be hoped that by giving early exposure to our CS students to the applications of CS to other disciplines, they will in the future be able to further their own discipline but also apply it to help solve the critical problems of our societies such as those of energy, environment, security, health care, or economics.

\section{References}

[1] Cozzens M B, Roberts F S (eds.). BioMath in the Schools DIMACS Series, Vol.76, American Mathematical Society, Providence, RI, 2011.

[2] Cozzens M B. New Materials to Integrate Mathematics and Biology in High School Curriculum. BioMath in the Schools, Cozzens M B, Roberts F S (eds.), DIMACS Series, Vol.76, American Mathematical Society, Providence, RI, 
2011, pp.97-108.

[3] Roberts F S. Why BioMath? Why Now? BioMath in the Schools. Cozzens M B, Roberts F S (eds.), DIMACS Series, Vol.76, American Mathematical Society, Providence, RI, 2011, pp.3-34.

[4] Levin S A, Clark W C. Toward a Science of Sustainability, Report from the Toward a Science of Sustainability Conference. CID Working Paper No.196, Airlie Centre, Warrenton, Virginia, Nov. 29-Dec. 2, 2009, http://www.nsf.gov.mps./ dms/documents/SustainabilityWorkshopReport.pdf.

[5] Cozzens M B, Roberts F S (eds.). Mathematical and Statistical Challenges for Sustainability. American Mathematical Society, Providence, RI, 2011.

[6] Bryant R, Fisher D, Gianchandani E, Gomes C, Rouse W, Shenoy P, Sproull R, Waltz D. Science, Engineering and Education of Sustainability: The Role of Information Sciences and Engineering. Computing Community Consortium, 2011, http://cra.org/ccc/docs/RISES_Workshop_Final_Report-510-2011.pdf.

[7] Roberts F S. Computer science and decision theory. Annals of Operations Research, 2008, 163: 209-253.

[8] Rossi F, Tsoukiàs A (eds.). Proceedings of ADT 2009, Venice, Italy, Oct. 20-23, 2009.

[9] Easley D, Kleinberg J. Networks, Crowds, and Markets. Cambridge University Press, 2010.

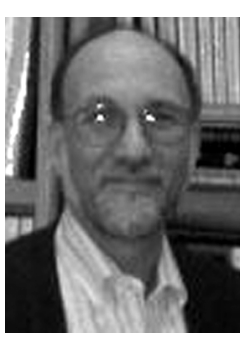

Fred S. Roberts is director of the Center for Discrete Mathematics and Theoretical Computer Science (DIMACS), Department of Homeland Security Center of Excellence CCICADA: Command, Control and Interoperability Center for Advanced Data Analysis. $\mathrm{He}$ is a professor in the Department of Mathematics, Rutgers University, a fellow of RUT-

COR, Rutgers Center for Operations Research, a member of Graduate Faculties in Mathematics, Operations Research, Computer Science, Computational Molecular Biology, Computational Biology and Molecular Biophysics, and Education. His research interests include mathematical models in the social, behavioral, biological and environmental sciences and of problems of communications and transportation; graph theory and combinatorics; measurement theory; operations research; utility, decisionmaking, and social choice; mathematical modeling and decisionmaking for homeland security. 